\title{
Complete unroofing of the intramural coronary artery for anomalous aortic origin of a coronary artery: The role of commissural resuspension?
}

\author{
Can Yerebakan, MD, ${ }^{\mathrm{a}}$ Mahmut Ozturk, MD, ${ }^{\mathrm{a}}$ Lucas Mota, BS, ${ }^{\mathrm{a}}$ Lok Sinha, MD, ${ }^{\mathrm{a}}$ \\ Heather Gordish-Dressman, PhD, ${ }^{\mathrm{b}}$ Richard Jonas, MD, ${ }^{\mathrm{a}}$ and Pranava Sinha, MD
}

\section{ABSTRACT}

Objectives: Although surgical repair of an anomalous aortic origin of the coronary artery has low operative mortality, longer-term risk of ischemia and aortic regurgitation remains concerning. We routinely perform aortic commissure resuspension after unroofing and sought to evaluate the outcomes with regard to aortic valve competence, symptoms, and signs of ischemia with this approach.

Methods: Twenty-six consecutive patients who received the unroofing procedure for anomalous aortic origin of the coronary artery (10 left; 16 right) between 2004 and 2016 were reviewed. In addition to complete unroofing of the intramural coronary, patients early in the cohort $(\mathrm{n}=9)$ received unroofing only, and aortic commissural resuspension was performed routinely in the subsequent patients $(\mathrm{n}=17)$. Outcomes between commissural resuspension versus no commissural resuspension were compared. The occurrence of mild and greater aortic regurgitation was assessed using a time-to-event analysis after varying lengths of time. Commissural resuspension was considered as the predictor, and the groups were compared using a log-rank test.

Results: There was no operative mortality. One patient in the no commissural resuspension group died 10 years later of prosthetic aortic valve endocarditis (aortic valve replacement 7 years after unroofing). The follow-up duration was 6.9 years (4.9-9.1) and 3.7 years (2.1-4.3) in the no commissural resuspension and commissural resuspension groups, respectively $(P=.001)$. Available postoperative exercise stress test data $(n=14)$ revealed that $50 \%$ had an endurance level at the 25 th percentile or greater for age. After a median follow-up of 1.9 years (3 months to 10.6 years), no patient in the commissural resuspension group had aortic regurgitation, whereas 6 of 9 patients $(67 \%)$ in the no commissural resuspension group had stable but mild or greater aortic regurgitation. Time-to-event analysis with the primary event of occurrence of mild or greater aortic regurgitation showed significantly higher freedom from the occurrence of aortic regurgitation in the commissural resuspension group $(P=.035)$.

Conclusions: Surgical repair of an anomalous aortic origin of the coronary artery can be performed with excellent early and midterm outcomes. Routine commissural resuspension of the aortic valve may lead to a lower rate of aortic valve regurgitation without increasing the risk of ischemia. ( $\mathrm{J}$ Thorac Cardiovasc Surg 2019;158:208-17)

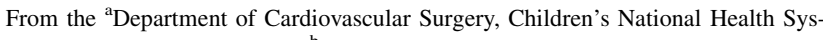
tem, Washington, DC; and ${ }^{b}$ Center for Genetic Medicine Research, Children's Health System, George Washington University School of Medicine and Health Sciences, Washington, DC.

Read at the 98th Annual Meeting of The American Association for Thoracic Surgery, San Diego, California, April 28-May 1, 2018.

Received for publication May 2, 2018; revisions received Jan 12, 2019; accepted for publication Jan 25, 2019; available ahead of print April 5, 2019.

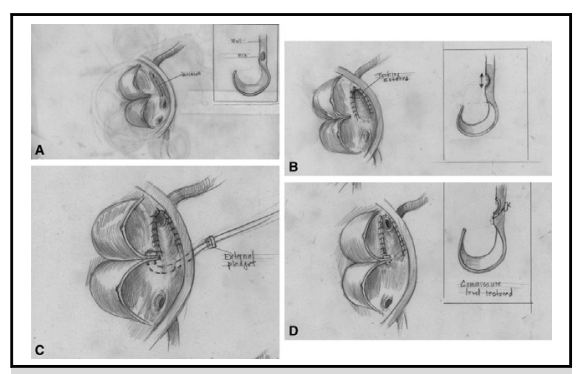

Repair of AAOLCA with CR.

\section{Central Message}

Routine aortic valve CR during the repair of an anomalous aortic origin of the coronary artery may improve long-term aortic valve function.

\section{Perspective}

Surgical repair of an anomalous aortic origin of a coronary artery can be accomplished with excellent operative results. Despite excellent short-term outcome, the patients continue to possess significant morbidity at longer-term follow-up. Aortic valve insufficiency is not a rare finding in the postoperative period in many published series. Our data indicate that routine $\mathrm{CR}$ of the adjacent aortic valve commissure may reduce the incidence of late aortic valve-related morbidity after repair.

See Commentary on page 218 . 

Abbreviations and Acronyms
$\begin{aligned} \mathrm{AAOCA}= & \text { anomalous aortic origin of coronary } \\ \text { artery } & \end{aligned}$
$\mathrm{AI}=$ aortic insufficiency
$\mathrm{CR}=$ commissural resuspension
IQR = interquartile range
NCR $=$ no commissural resuspension
SCD = sudden cardiac death

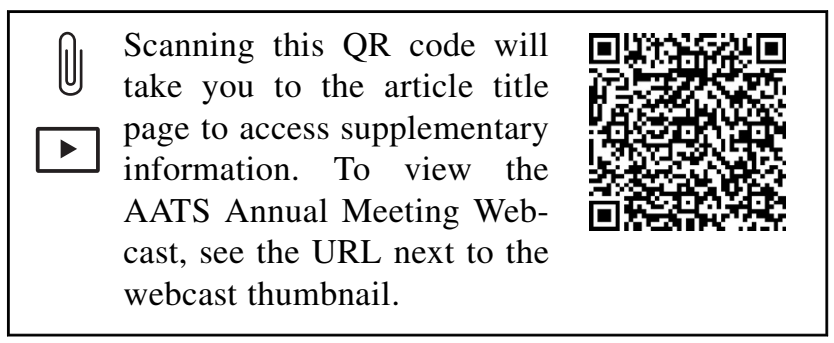

Anomalous aortic origin of coronary artery (AAOCA) is a rare coronary anomaly that may be associated with an intramural or interarterial course. Reports from angiographic evaluations indicate an incidence of up to $1.2 \%$, with involvement of the right coronary artery more common than the left coronary artery ${ }^{2,3}$; however, the latter carries a higher risk. The pathology is reported to be the second most common reason for sudden cardiac death (SCD) in young athletes. ${ }^{4}$ The risk stratification of patients with AAOCA remains challenging because of the impact of anatomic variations, patient factors, ${ }^{5,6}$ absence of symptoms in the majority of patients, and SCD representing a small proportion of this group, ${ }^{7}$ leading to a wide variation in management of these patients between institutions. ${ }^{8}$ Despite excellent surgical survival, symptoms and morbidity postoperatively after AAOCA repair, ${ }^{9-11}$ and even major cardiac events such as SCD have been observed postoperatively. ${ }^{12,13}$ An important outcome measure after this operation is the function of the aortic valve, and progressive aortic regurgitation remains an important indication for continued postoperative morbidity and need for reintervention. ${ }^{13}$

Surgical repair usually consists of unroofing of the intramural segment and neo-ostium creation using intimal reapproximation. Although aortic valve commissure resuspension is standard in patients who require commissural detachment for the unroofing, it is not routinely performed in other cases. ${ }^{14}$ After our initial experience, we routinely perform aortic valve resuspension in all cases irrespective of whether commissural detachment is needed for unroofing or not. This study aims to evaluate our experience in patients with AAOCA in a medium-term follow-up, particularly with respect to postoperative aortic valve function with routine aortic valve resuspension.

\section{MATERIALS AND METHODS Study Population}

All patients who underwent a surgical repair for right or left AAOCA and intramural course between 2004 and 2016 at our center were included. Because the study aimed at evaluation of aortic valve function in the midterm follow-up, patients with postoperative follow-up less than 6 months were excluded from the main analysis. A separate actuarial time to event analysis for all patients (including the patients with shorter follow-up) was also done to allow complete data reporting and is added as a Supplemental file. Institutional Review Board approved waiver of informed consent for this retrospective study.

\section{Preoperative Management}

Preoperative investigations included transthoracic echocardiography, electrocardiogram, stress testing, and computed tomography or cardiac magnetic resonance imaging. Standard intraoperative transesophageal echocardiography was performed in all patients. All symptomatic patients and all patients with left AAOCA with an intramural course were offered surgery. In asymptomatic patients with right AAOCA, surgery was offered if preoperative imaging showed a narrowed coronary ostium or long intramural segment, with due consideration given to patients' and families' perspectives on medical management with activity restrictions after a full discussion of the surgical risks.

\section{Surgical Technique}

Via a full median sternotomy, moderate hypothermia, aortic and 2 -stage venous cannulation was used for cardiopulmonary bypass, along with antegrade cardioplegia administration and reverse hockey stick aortotomy for exposure. Two patients had their procedures done via an upper ministernotomy. The surgical technique included a complete unroofing of the intramural segment of the anomalous coronary artery in all cases. This involved unroofing of the entire intramural coronary course past the level of the aortic valve commissure up to the level where the coronary artery exits the aorta. Intimal tacking along the unroofing incision was performed using continuous 6-0 sutures. In the early years, aortic valve resuspension was performed at the surgeon's discretion; however, later on, all patients routinely received resuspension of the aortic valve commissure. By unroofing the intramural segment, the decrease in the level of the related commissure is equal to the radius of the intramural vessel (Figures E1-E3). This is easily reversed by reattaching the commissure at exactly at the midpoint of the unroofed intramural segment using a 5-0 Prolene suture passed through the aorta and tied externally over a pledget (Figure 1). A small internal pericardial pledget was used early in our experience. However, later in our experience, we do not use any internal pledgets because the tissue properties of the commissural area and how well it holds sutures make it unnecessary (Video 1). In patients with shorter intramural segments, moving the resuspension suture 1 to $2 \mathrm{~mm}$ away from the neo-ostium ensures an uncompromised blood flow to the coronary artery.

\section{Postoperative Management}

Patients follow the fast-tract intensive care protocol with early extubation, postoperative pain management, ambulation, and physical therapy. Aspirin $(325 \mathrm{mg}$ ) is administered orally or via a nasogastric tube within 6 hours of intensive care unit admission followed by $81 \mathrm{mg}$ oral dose daily. Patients undergo exercise stress testing at 3 to 6 months postoperatively, after which activity restrictions are removed and aspirin may be stopped. 

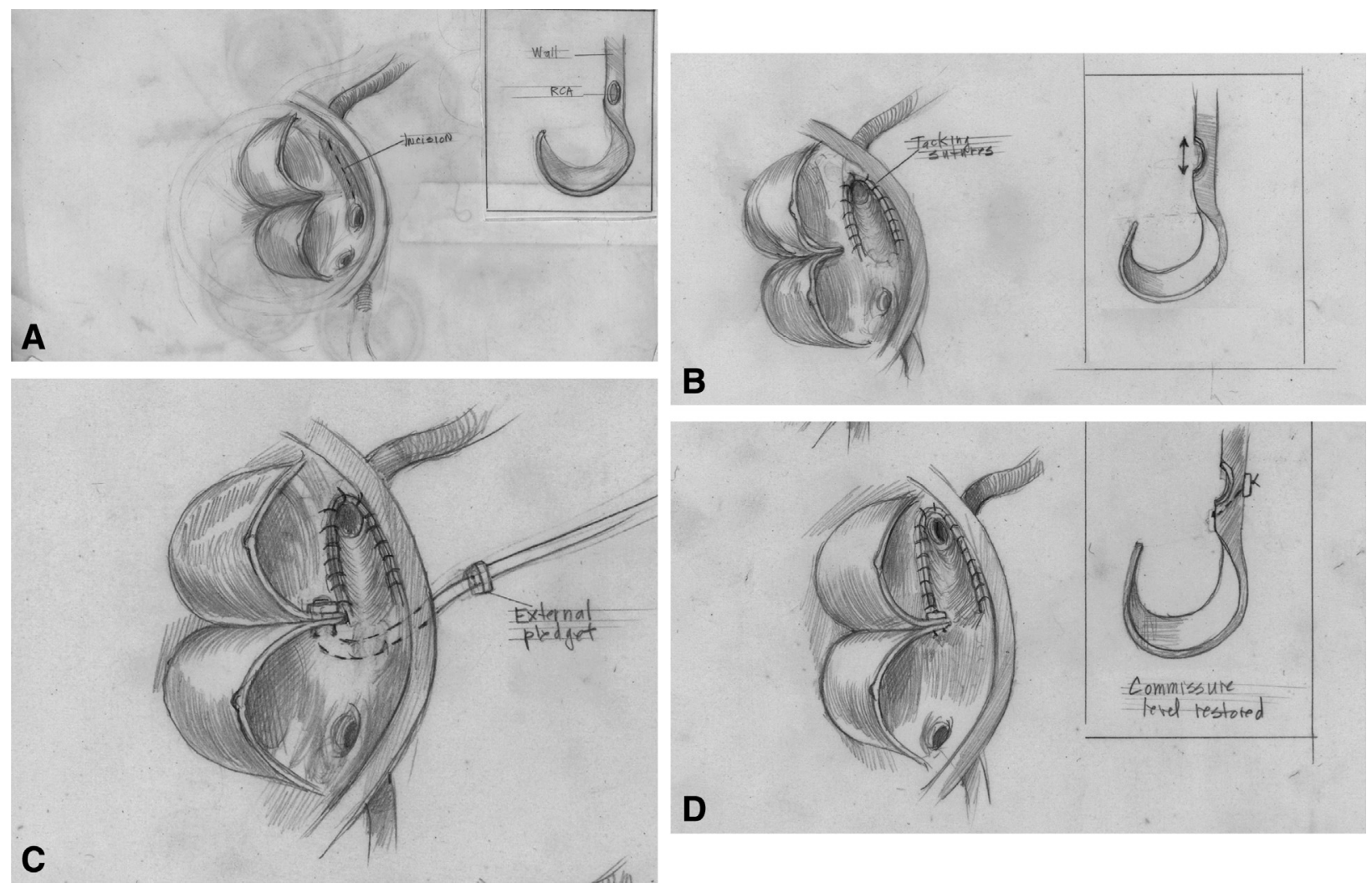

B

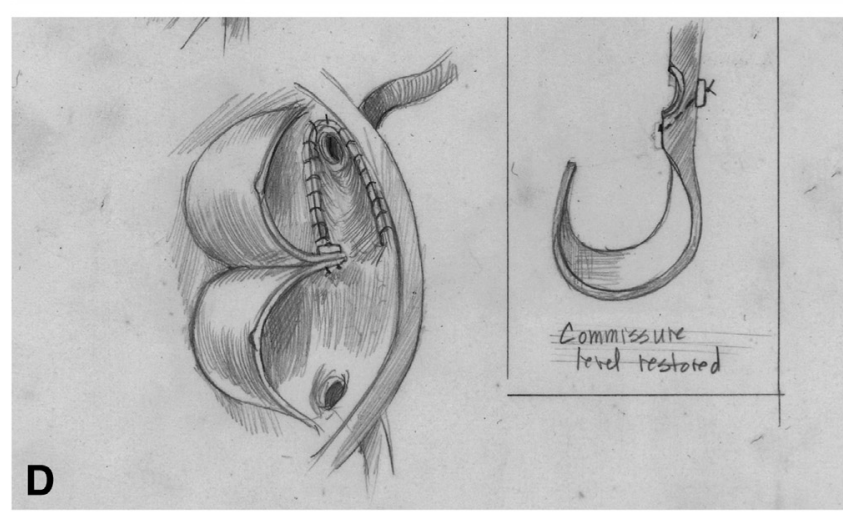

FIGURE 1. A, Anomalous left coronary artery from the right sinus of Valsalva. Inset shows intramural course. B, Unroofing and intimal tacking. Inset shows commissural dropdown equal to the half of the diameter of the intramural segment upon incision of the common wall. $\mathrm{C}$, CR of the aortic valve commissure using monofilament pledged suture. D, Final result. Commissure restored to the baseline level by attachment halfway up along the unroofed intramural segment. For shorter intramural coronary length, the commissure may be moved 1 to $2 \mathrm{~mm}$ away from the neo-ostium to ensure uncompromised flow to the coronary artery. RCA, Right coronary artery.

\section{Data Collection}

Data were obtained via retrospective review of patient charts. Baseline demographic data, indications for surgery, and type of surgical repair were recorded. We evaluated the early and late postoperative outcomes with regard to survival, major cardiac events, reinterventions, aortic valve function, surgical complications, electrocardiogram changes, stress test evaluation, and symptoms.

Estimation of aortic insufficiency (AI) grade was standard for all echocardiographic evaluations and performed using an integrative and comprehensive process based on all objective information collected during the echocardiographic examination as recommended by the American Society of Echocardiography. ${ }^{15}$

\section{Statistical Analysis}

Categorical data are presented as numbers (proportions), and continuous data are presented as median (interquartile range [IQR]) or mean (standard deviation) where appropriate. Intergroup comparison (commissural resuspension [CR] vs no commissural resuspension [NCR] groups) of preoperative and postoperative variables were compared using univariate analysis with the Fisher exact test for categorical variables and $t$ test or Kruskal-Wallis test for continuous variables.

The length of the intramural segment was not included in the analysis because only 16 of 26 patients had the data documented intraoperatively. Cardiopulmonary bypass time and crossclamp times were not included because there was no intergroup difference between the CR and NCR groups.

The occurrence of mild and greater aortic regurgitation was assessed using a time-to-event analysis after varying lengths of time using KaplanMeier analysis and log-rank test to compare the CR and NCR groups.

\section{RESULTS}

A total of 26 consecutive patients with postoperative follow-up greater than 6 months were included in the study. Median age was 15.5 years (IQR, 10.2-17.7). Sixteen patients had anomalous left coronary artery, and 10 patients had anomalous right coronary artery. In the entire group, 20 patients were symptomatic preoperatively, among whom 2 patients were resuscitated after a cardiac arrest, 5 patients had syncopal episodes, and 13 patients presented with exertional dyspnea.

One patient who had prior cardiac arrest had moderate to severe depression of the left ventricular systolic function preoperatively. All other patients had normal left ventricular function. No patient had any aortic regurgitation preoperatively. The surgical repair consisted of unroofing with 


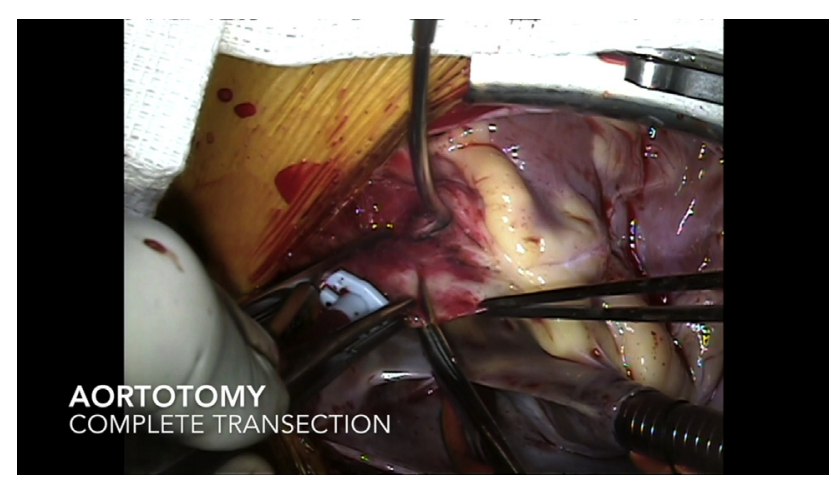

VIDEO 1. The coronary unroofing procedure in a 6-year-old male patient with an anomalous right coronary artery from the left sinus of Valsalva is shown. After complete transection of the ascending aorta and anatomic inspection, a commissural stich with 5/0 Prolene suture was placed. The anomalous right coronary was unroofed completely. The intima was tacked with a continuous 6/0 Prolene suture, creating a neo-ostium. The left-right commissure was resuspended using a 5/0 Prolene suture, which was reinforced with a felt pledget outside of the aorta. The aortic coaptation and commissural height were assessed by direct vision and transesophageal echocardiography. Video available at: https://www.jtcvs.org/article/ S0022-5223(19)30537-9/fulltext.

NCR in 9 earlier patients (NCR group) and unroofing with $\mathrm{CR}$ in 17 patients (CR group).

The groups were comparable with regard to age, weight, left or right coronary involvement, symptoms, ventricular function, cardiopulmonary bypass and aortic crossclamp times, and postoperative outcomes. There were no operative deaths. The length of the intramural segment had been documented in the operative records in only 16 patients.

The median (IQR) follow-up of the entire cohort was 4.3 (3.5-5) years. The follow-up duration was significantly longer in the NCR group (median [IQR]; NCR group, 6.9 [4.9-9.1] years vs $\mathrm{CR}$ group 3.7 [2.1-4.3] years $[P=.001])$. One patient died 11 years after the coronary unroofing. This patient had progressive postoperative aortic regurgitation, underwent a mechanical aortic valve replacement 7 years later, and died of aortic valve endocarditis and severe left ventricular failure 11 years after the index operation. No other patients required any reinterventions. No patients had any postoperative symptoms. The preoperative, intraoperative, and postoperative details are summarized in Table 1.

\section{Aortic Valve Function}

None of the patients had aortic regurgitation preoperatively. In the immediate postoperative period, 5 patients had mild aortic regurgitation (4 in the NCR group, 1 in the $\mathrm{CR}$ group) and 1 patient had progressive late severe aortic regurgitation (NCR group) (CR vs NCR group, $P=.009$ ).

Follow-up echocardiograms were available in $100 \%$ of the patients. Studies performed after a median (IQR) postoperative period of $1.02(0.04-2.4)$ years (NCR group, 2.4 [0.03-6.9] years vs CR group, 0.8 [0.06-1.8] years, $P=.2$ ) revealed that all patients in the $\mathrm{CR}$ group had no or trivial aortic regurgitation, whereas 6 of 9 patients in the NCR group had stable but mild or greater aortic regurgitation $(P<.001)$ (Table 2). The immediate and late follow-up echocardiographic findings are summarized in Table 2 and Figure 2.

Kaplan-Meier analysis and log-rank test demonstrated a significantly greater freedom from mild of greater aortic regurgitation in patients who had CR (Figure 3). Figure E4 shows the Kaplan-Meier analysis that also includes patients with less than 6 months follow-up who were excluded from the main analysis. Again, there was significantly greater freedom from mild or greater aortic valve regurgitation in patients who had $\mathrm{CR}$.

\section{Stress Test}

No patient was on exercise restriction. Exercise stress testing data were available in 11 patients $(\mathrm{NCR}=4$, $\mathrm{CR}=7)$. Exercise stress testing was performed after a median (IQR) of 112.5 (111-114) days (NCR group, 110 [108.9-110.6] days vs CR group, 113.3 [112.5-114.4] days, $P=.01)$. Although there was no difference between the CR and NCR groups $(P=.24)$, overall 5 patients $(45 \%)$ had an endurance level at or below the 25 th percentile for age. One patient demonstrated ST changes at exercise stress test without any symptoms. Follow-up imaging in this patient revealed no coronary stenosis (Table 3).

\section{Comments}

The guidelines and current practices for the surgical treatment of an AAOCA are still evolving. The indication for a surgical repair in AAOLCA and symptomatic patients with AAORCA is evident; indication for intervention in asymptomatic AAORCA differs between institutions. Likewise, surgical techniques tend to differ with respect to length of intramural unroofing, incision versus excision of the common wall, with or without intimal tacking. A recent report of patients in the Congenital Heart Surgeons' Society AAOCA Registry of 101 intramural coronary artery unroofing procedures showed that aortic valve resuspension was performed in only 33 patients $(32 \%) .{ }^{14}$ Immediate surgical outcomes for the repair of AAOCA are excellent with regard to survival. However, patients are known to have persistent symptoms, and a higher number of midterm and longer-term reports indicate a considerable rate of morbidity after the repair of an AAOCA. ${ }^{10,13,15,16}$

An important outcome measure after this operation is the function of the aortic valve, and progressive aortic regurgitation remains an important indication for continued postoperative morbidity and need for reintervention. The incidence of mild or greater aortic valve regurgitation is as high as $12 \%$ to $17 \%$ in some series, leading to 
TABLE 1. Clinical characteristics of patients with anomalous aortic origin coronary artery: Preoperative, intraoperative, and postoperative data

\begin{tabular}{|c|c|c|c|c|}
\hline \multirow[b]{2}{*}{ Variable } & \multicolumn{4}{|c|}{ Preoperative variables } \\
\hline & $\operatorname{NCR}(\mathbf{n}=9)$ & CR $(n=17)$ & All $(\mathbf{N}=\mathbf{2 6})$ & $P$ value \\
\hline Age, y & $15.4(13.9-16.9)$ & $15.8(10.1-17.9)$ & $15.5(10.2-17.7)$ & .53 \\
\hline Weight, kg & $58.3 \pm 27.6$ & $57.4 \pm 20.9$ & $57.7 \pm 22.8$ & .9 \\
\hline $\begin{array}{l}\text { Coronary artery involved } \\
\text { Left } \\
\text { Right }\end{array}$ & $\begin{array}{l}4(44 \%) \\
5(55 \%)\end{array}$ & $\begin{array}{r}12(71 \%) \\
5(29 \%)\end{array}$ & $\begin{array}{l}16(61.5 \%) \\
10(38.5 \%)\end{array}$ & .23 \\
\hline $\begin{array}{l}\text { Presence of symptoms } \\
\text { Syncope } \\
\text { Cardiac arrest } \\
\text { Exertional dyspnea }\end{array}$ & $\begin{array}{l}1(11 \%) \\
1(11 \%) \\
5(55 \%)\end{array}$ & $\begin{array}{l}4(23 \%) \\
1(6 \%) \\
8(47 \%)\end{array}$ & $\begin{array}{c}5(19.2 \%) \\
2(7.7 \%) \\
13(50 \%)\end{array}$ & 1.0 \\
\hline Preoperative AI & 0 & 0 & 0 & \\
\hline Preoperative LV dysfunction & $1(11 \%)$ & 0 & $1(3 \%)$ & .34 \\
\hline \multicolumn{5}{|c|}{ Intraoperative and postoperative variables } \\
\hline $\begin{array}{l}\text { Length of intramural } \\
\text { segment, } \mathrm{mm}^{*}\end{array}$ & $6.8 \pm 1$ & $6.2 \pm 1.8$ & $6.3 \pm 1.5$ & .5 \\
\hline CPB time, $\min$ & $54(49-90)$ & $56(49-70)$ & $55(49-71.5)$ & .68 \\
\hline ACC time, min & $30(29-50)$ & $39(29-49)$ & $35.5(29-49.8)$ & .75 \\
\hline Ventilation time, $\mathrm{h}$ & $8(7-13)$ & $6(5-10)$ & $7.6(5-10.4)$ & .22 \\
\hline ICU LOS stay, d & $1(1-1.25)$ & $2(1-4)$ & $2(1-2)$ & .06 \\
\hline $\begin{array}{l}\text { Postoperative hospital LOS, } \\
\quad \mathrm{d}\end{array}$ & $5(4-5)$ & $4(4-4)$ & $4(4-5)$ & .35 \\
\hline Operative mortality & 0 & 0 & 0 & \\
\hline Clinical follow-up, y & $6.9(4.9-9.1)$ & $3.7(2.1-4.3)$ & $4.3(3.5-5)$ & $.001 \dagger$ \\
\hline Late LV dysfunction & $1(11 \%)$ & 0 & $1(3 \%)$ & .34 \\
\hline Reoperation & $1(11 \%)$ & 0 & $1(3 \%)$ & .34 \\
\hline Late mortality & $1(11 \%)$ & 0 & $1(3 \%)$ & .34 \\
\hline
\end{tabular}

$\overline{N C R}$, No commissural resuspension; $C R$, commissural resuspension; $A I$, aortic valve insufficiency; $L V$, left ventricle; $C P B$, cardiopulmonary bypass; $A C C$, aortic crossclamp; $I C U$, intensive care unit; $L O S$, length of stay. ${ }^{*}$ The intramural length was measured in only 16 patients. Categorical data presented as numbers $(\%)$, and continuous data presented as median (IQR) or mean \pm standard deviation. CR group compared with NCR group using Fisher exact test for categorical data and $t$ test or Kruskal-Wallis test for continuous data. $†$ Statistically significant difference between NCR versus $C R$ groups.

progressive and severe aortic valve regurgitation and occasionally the requirement for late aortic valve replacement. ${ }^{9,13,17}$ Nees and colleagues ${ }^{13}$ reported an incidence of postoperative $\mathrm{AI}$ in 7 of 56 patients $(12 \%)$, with 1 of them needing aortic valve replacement. Likewise, Fabozzo and colleagues ${ }^{9}$ reported a $3 \%$ need for reintervention in their series because of aortic regurgitation, but the number of patients with postoperative aortic regurgitation was not reported. It seems that reintervention/reoperation is a less sensitive index and may be underestimating the aortic valve-related complication of the unroofing procedure. Therefore, as do Nees and colleagues ${ }^{13}$ we choose echocardiographic evidence of mild or greater $\mathrm{AI}$ as an end point, which is a more sensitive indicator of any change in the aortic valve function.

The aortic valve commissures are always in close proximity to the intramural coronary course. In the rare instances when the intramural segment of the anomalous coronary artery is below the top of the related commissure, commissural takedown has to be performed to achieve unroofing. However, even in patients with the intramural segment above the commissure, unroofing the intramural segment leads to lowering of the commissure. This drop in the commissure is equal to the radius of the intramural vessel diameter. The height of the commissure can be easily restored by reattaching the commissure at exactly the midpoint of the unroofed intramural segment. In patients with shorter intramural segments, moving the resuspension suture 1 to $2 \mathrm{~mm}$ away from the neo-ostium ensures uncompromised flow to the coronary artery.

After our early experience with the unroofing procedure, we have resorted to CR in every case, irrespective of the proximity of the intramural vessel to the commissure and whether commissural takedown is required. We also believe that complete unroofing of the intramural coronary segment is imperative in achieving a completely unobstructed 
TABLE 2. Comparison of echocardiographic data on aortic valve function at follow-up between the 2 groups

\begin{tabular}{|c|c|c|c|c|}
\hline Variable & $\operatorname{NCR}(\mathbf{n}=9)$ & $\mathbf{C R}(\mathbf{n}=17)$ & All $(\mathbf{N}=\mathbf{2 6})$ & $P$ value \\
\hline \multicolumn{5}{|c|}{ Aortic regurgitation in the immediate postoperative period } \\
\hline No or trivial AI & $4(44 \%)$ & $16(94 \%)$ & $20(76.9 \%)$ & $.009 *$ \\
\hline Mild AI & $4(44 \%)$ & $1(6 \%)$ & $5(19.2 \%)$ & \\
\hline Moderate to severe AI & $1(11 \%)$ & 0 & $1(3.8 \%)$ & \\
\hline \multicolumn{5}{|c|}{ Aortic regurgitation at last follow-up } \\
\hline No or trivial AI & $3(33 \%)$ & $17(100 \%)$ & $20(76.9 \%)$ & \\
\hline Mild AI & $3(33 \%)$ & 0 & $3(11.6 \%)$ & \\
\hline Moderate to severe AI & $3(33 \%)$ & 0 & $3(11.6 \%)$ & \\
\hline Echocardiographic & $2.4(0.03-6.9)$ & $0.8(0.06-1.8)$ & $1.02(0.04-2.4)$ & .2 \\
\hline Follow-up period, y & & & & \\
\hline
\end{tabular}

Categorical data presented as numbers (\%), and continuous data presented as median (IQR). CR group compared with NCR group using Fisher exact test for categorical data and Kruskal-Wallis test for continuous data. NCR, No commissural resuspension; $C R$, commissural resuspension; $A I$, aortic valve insufficiency. *Statistically significant difference between NCR versus CR groups.

neostium and preventing postoperative stenosis, which may account for some of the residual symptoms and signs and anatomic findings of restenosis. As a result, we unroof the entire intramural segment up to the point where the coronary exits the aorta, followed by intimal tacking for neo-ostial reconstruction. In patients with short intramural segments, a coronary reimplantation or pulmonary artery reconstruction may be required to prevent compression by the intracoronary commissure. We did not use these techniques in this cohort.

Our study with limited follow-up shows that routine CR helps to maintain aortic valve competence. No patient in our group with CR showed more than trivial aortic regurgitation in the follow-up, whereas when CR was not performed, $55 \%$ of the patients had mild or greater aortic regurgitation, which progressed to involve $66 \%$ over the median followup time of 2.4 years. The only reintervention in our series was in 1 patient in the NCR group, who required an aortic valve replacement 7 years after the unroofing procedure and subsequently died of complications of prosthetic valve endocarditis 11 years later.

In our series of patients, all of whom underwent complete unroofing, no patient had postoperative cardiac events or symptoms. On exercise stress testing, only 1 patient had a suggestion of ischemia; however, anatomic imaging revealed no coronary stenosis.

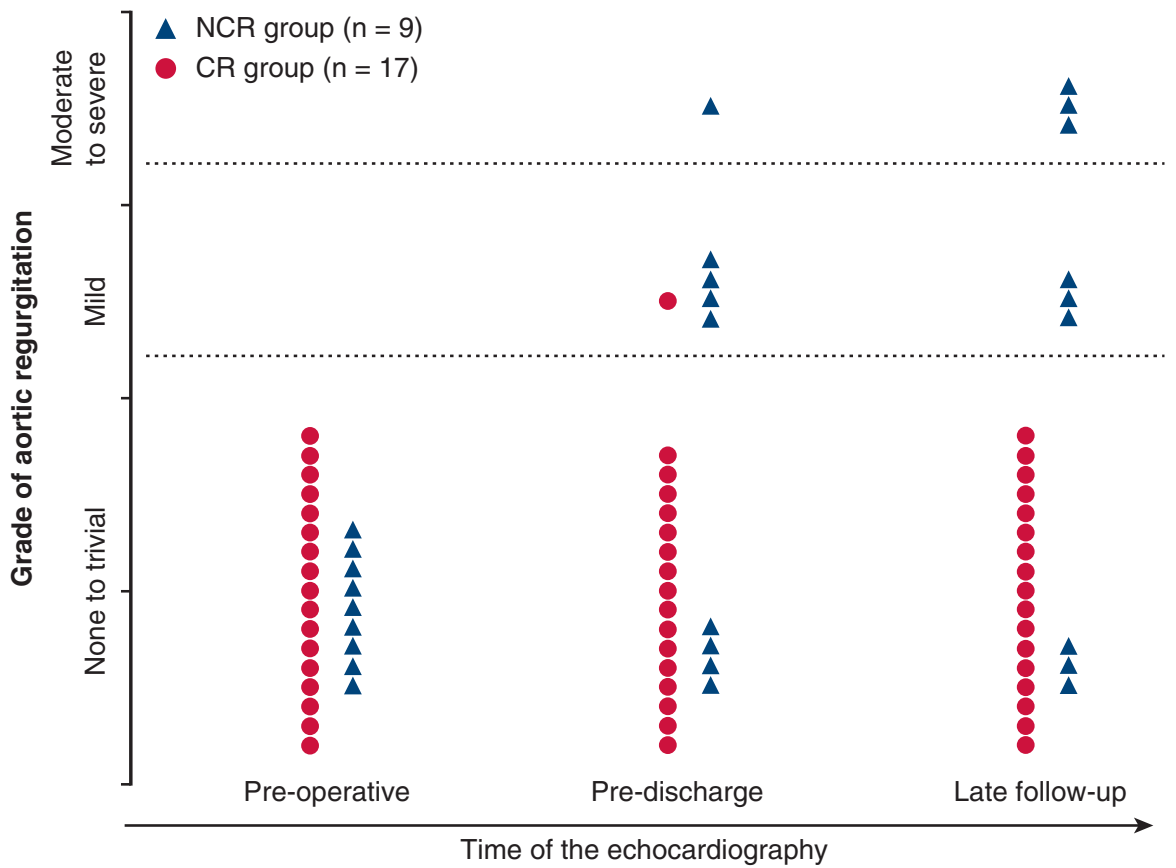

FIGURE 2. The grade of AI (y-axis) over the follow-up in comparison with the preoperative level in the CR and NCR groups at 3 time points preoperatively, predischarge, and later follow-up ( $x$-axis). Green dots: CR group. Red triangles: NCR group. $C R$, Commissural resuspension; NCR, no commissural resuspension. 


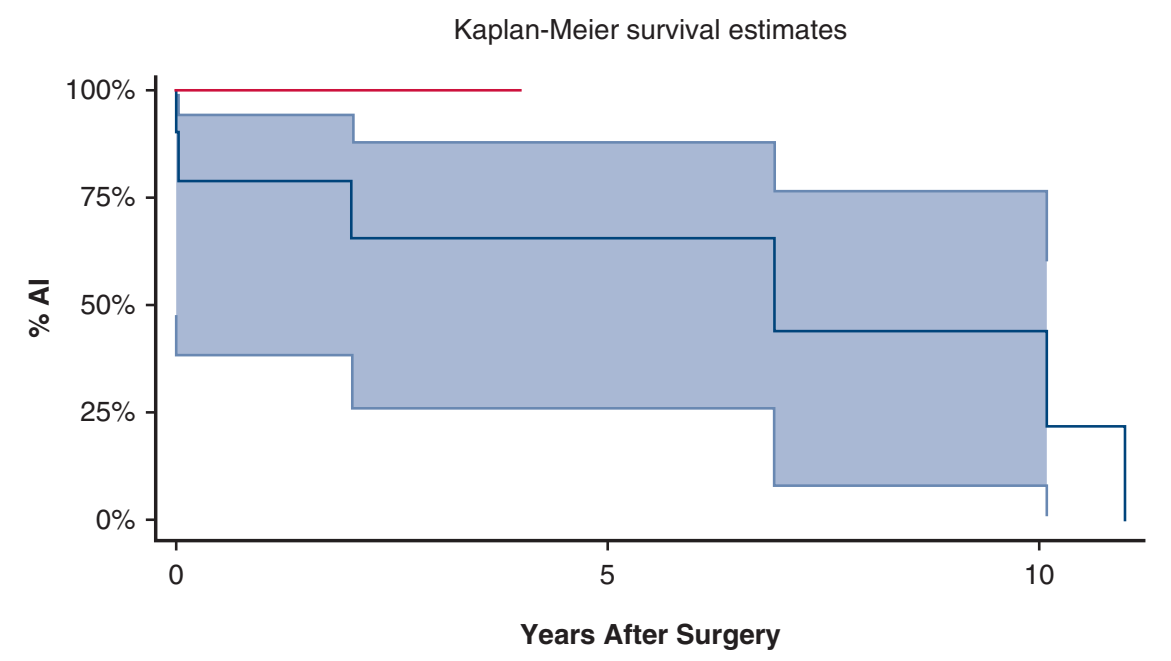

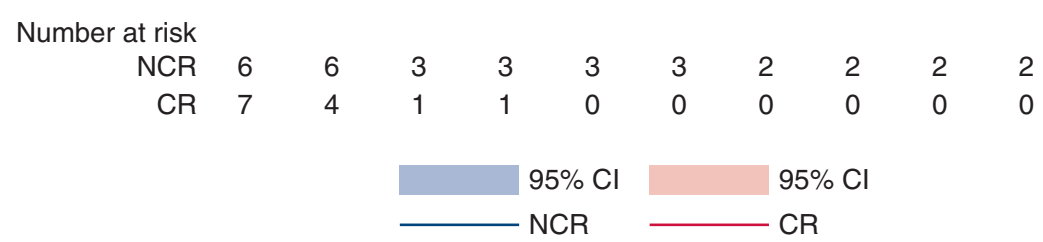

FIGURE 3. Kaplan-Meier curves and $95 \%$ confidence intervals showing freedom from mild or greater aortic regurgitation in patients with follow-up $\geq 6$ months. A log-rank test $(P=.035)$ demonstrated a greater freedom from mild or greater aortic regurgitation in patients who had a CR (CR group) compared with those who did not (NCR group). Patients without aortic regurgitation were censored the time of last known echocardiographic examination. $A I$, Aortic insufficiency; $C I$, confidence interval; $C R$, commissural resuspension; NCR, no commissural resuspension.

The prevention of SCD is the main indication for the operation. Most patients with this disease are advised activity restriction preoperatively. The postoperative stress testing in our cohort was done after a median of 112 days after surgery before lifting exercise restriction. As expected, most of these patients were deconditioned as shown by approximately half of our patients performing at less than the 25th percentile of expected exercise capacity. As previously reported, the longer-term exercise capacity and quality of life in these patients are expected to be normal. ${ }^{16,18}$ No patient in our series had symptoms or signs of ischemia. Only 1 patient had ST-segment changes on exercise stress testing, but imaging was negative for any anatomic coronary artery stenosis. This suggests that complete unroofing of the intramural coronary with routine $\mathrm{CR}$ does not increase postoperative risk for ischemia.

\section{Study Limitations}

Our study has the limitation of being a single-center retrospective study of a small patient cohort with a short follow-up duration. The median clinical follow-up between the CR and NCR groups is different with shorter follow-up time for the CR group. Postoperative clinical follow-up data were analyzed, and exercise stress testing was done on a treadmill using the standard Bruce protocol. A longer follow-up of a larger group of patients using exercise stress imaging for functional and anatomic data is needed to validate the outcomes of this study. The number of patients is

TABLE 3. Comparison of exercise stress test finding between the 2 groups

\begin{tabular}{|c|c|c|c|c|}
\hline Variable & $\operatorname{NCR}(n=4)$ & $\mathbf{C R}(\mathbf{n}=7)$ & All $(\mathbf{n}=11)$ & $P$ value \\
\hline \multicolumn{5}{|l|}{ Exercise capacity } \\
\hline $0-25$ th percentile & $3(75 \%)$ & $2(28 \%)$ & $5(45 \%)$ & .24 \\
\hline 26-50th percentile & $1(25 \%)$ & $1(14 \%)$ & $2(18 \%)$ & \\
\hline 51-75th percentile & 0 & $1(14 \%)$ & $1(9 \%)$ & \\
\hline 75-100th percentile & 0 & $3(42 \%)$ & $3(27 \%)$ & \\
\hline ST elevation & 0 & $1(14 \%)$ & $1(9 \%)$ & 1.0 \\
\hline Exercise stress test follow-up period, $d$ & $110(108.9-110.6)$ & $113.3(112.5-114.4)$ & $112.5(111-114)$ & $.01 *$ \\
\hline
\end{tabular}

Categorical data presented as numbers (\%), and continuous data presented as median (IQR). CR group compared with NCR group using Fisher exact test for categorical data and Kruskal-Wallis test for continuous data. $N C R$, No commissural resuspension; $C R$, commissural resuspension. *Statistically significant difference between NCR versus CR groups. 
still too low to draw strong conclusions from the existent data because of limitations in the statistical analysis. Interobserver bias in estimation of AI grade was minimized by using a standard for all echocardiographic evaluations and performed using an integrative and comprehensive process based on all objective information collected during the echocardiographic examination as recommended by the American Society of Echocardiography. ${ }^{15}$

However, our data suggest that routine CR helps to maintain the competence of the aortic valve and reduces postoperative aortic regurgitation. Complete unroofing of the entire intramural segment may reduce postoperative symptoms, restenosis, and cardiac events.

\section{CONCLUSIONS}

Surgical repair of an AAOCA can be performed with excellent outcomes. Routine CR of the aortic valve may lead to a lower rate of aortic valve regurgitation without increasing the risk of ischemia.

\section{Webcast}

You can watch a Webcast of this AATS meeting presentation by going to: https://aats.blob.core.windows.net/ media/18May01/24ABC\%202.Congenital\%20SS/S87\%20\%20Part\%201/S87_3_webcast_023023762.mp4.
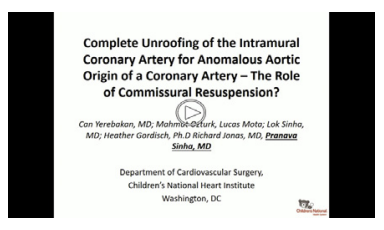

\section{Conflict of Interest Statement}

Authors have nothing to disclose with regard to commercial support.

\section{References}

1. Yuksel S, Meric M, Soylu K, Gulel O, Zengin H, Demircan S, et al. The primary anomalies of coronary artery origin and course: a coronary angiographic analysis of 16,573 patients. Exp Clin Cardiol. 2013;18:121-3.

2. Davis JA, Cecchin F, Jones TK, Portman MA. Major coronary artery anomalies in a pediatric population: incidence and clinical importance. J Am Coll Cardiol. 2001;37:593-7.

3. Agrawal H, Mery CM, Krishnamurthy R, Molossi S. Anatomic types of anomalous aortic origin of a coronary artery: a pictorial summary. Congenit Heart Dis. 2017; 12:603-6.

4. Maron BJ, Doerer JJ, Haas TS, Tierney DM, Mueller FO. Sudden deaths in young competitive athletes analysis of 1866 deaths in the United States, 1980-2006. Circulation. 2009;119:1085-92.

5. Brothers JA, Frommelt MA, Jaquiss RDB, Myerburg RJ, Fraser CD, Tweddell JS. Expert consensus guidelines: anomalous aortic origin of a coronary artery. $J$ Thorac Cardiovasc Surg. 2017;143:1440-57.

6. Van Hare GF, Ackerman MJ, Evangelista JAK, Kovacs RJ, Myerburg RJ, Shafer KM, et al. Eligibility and disqualification recommendations for competitive athletes with cardiovascular abnormalities: task force 4: congenital Heart disease: a Scientific Statement from the American Heart Association and American College of Cardiology. J Am Coll Cardiol. 2015;66:2372-84.
7. Mainwaring RD. "Cui periculum"-Who is at risk? J Thorac Cardiovasc Surg. 2018;155:322-4.

8. Agrawal H, Mery CM, Day PE, Sexson Tejtel SK, McKenzie ED, Fraser CD Jr, et al. Current practices are variable in the evaluation and management of patients with anomalous aortic origin of a coronary artery: results of a survey. Congenit Heart Dis. 2017:12:610-4.

9. Fabozzo A, DiOrio M, Newburger JW, Powell AJ, Liu H, Fynn-Thompson F, et al. Anomalous aortic origin of coronary arteries: a single-center experience. Semin Thorac Cardiovasc Surg. 2016;28:791-800.

10. Wittlieb-Weber CA, Paridon SM, Gaynor JW, Spray TL, Weber DR, Brothers JA Medium-term outcome after anomalous aortic origin of a coronary artery repair in a pediatric cohort. J Thorac Cardiovasc Surg. 2014;147:1580-6.

11. Frommelt PC, Sheridan DC, Berger S, Frommelt MA, Tweddell JS. Ten-year experience with surgical unroofing of anomalous aortic origin of a coronary artery from the opposite sinus with an interarterial course. J Thorac Cardiovasc Surg. 2011;142:1046-51.

12. Agrawal H, Sexson-Tejtel SK, Qureshi AM, Alam M, Masand P, Fraser CD Jr et al. Aborted sudden cardiac death after unroofing of anomalous left coronary artery. Ann Thorac Surg. 2017;104:e265-7.

13. Nees SN, Flyer JN, Chelliah A, Dayton JD, Touchette L, Kalfa D, et al. Patients with anomalous aortic origin of the coronary artery remain at risk after surgical repair. J Thorac Cardiovasc Surg. 2018;155:2554-64.e3.

14. Poynter JA, Bondarenko I, Austin EH, DeCampli WM, Jacobs JP, Ziemer G, et al Repair of anomalous aortic origin of a coronary artery in 113 patients: a Congenital Heart Surgeons' Society Report. World J Pediatr Congenit Heart Surg. 2014;5:507-14.

15. Zoghbi WA, Enriquez-Sarano M, Foster E, Grayburn PA, Kraft CD, Levine RA et al; American Society of Echocardiography. Recommendations for evaluation of the severity of native valvular regurgitation with two-dimensional and Doppler echocardiography. J Am Soc Echocardiogr. 2003;16:777-802.

16. Brothers JA, McBride MG, Seliem MA, Marino BS, Tomlinson RS, Pampaloni MH, et al. Evaluation of myocardial ischemia after surgical repair of anomalous aortic origin of a coronary artery in a series of pediatric patients. J Am Coll Cardiol. 2007;50:2078-82.

17. Romp RL, Herlong JR, Landolfo CK, Sanders SP, Miller CE, Ungerleider RM, et al. Outcome of unroofing procedure for repair of anomalous aortic origin of left or right coronary artery. Ann Thorac Surg. 2003;76:589-96.

18. Brothers JA, McBride MG, Marino BS, Tomlinson RS, Seliem MA, Pampaloni MH, et al. Exercise performance and quality of life following surgical repair of anomalous aortic origin of a coronary artery in the pediatric population. J Thorac Cardiovasc Surg. 2009;137:380-4.

Key Words: aorta, coronary anomaly, surgical repair, unroofing, myocardial ischemia, aortic valve

\section{Discussion}

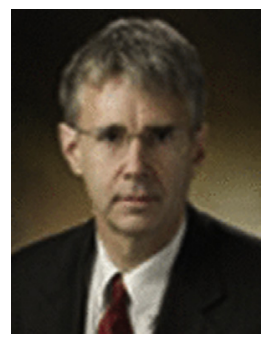

Dr J. Gaynor (Philadelphia, Pa). As we have heard this morning, most of the children who undergo an unroofing for anomalous aortic origin of the coronary are asymptomatic, and we are essentially performing a prophylactic operation. So in that case, both the shortand long-term safety have to be of utmost importance, and unfortunately as we have heard and as you suggested here, late competence of the aortic valve cannot always be guaranteed with the standard operation.

You and your co-authors have described a simple technical modification that seems to result in improved intermediateterm competence of the aortic valve, and I think this is a good thing and probably something that, again, as you suggest, needs to be studied in a larger cohort but seems to be a simple modification that may improve long-term function. 
Even when you unroof, I have seen the change in the commissural post position even if you don't have to detach the commissures. So there is plausibility why this would improve function.

I have 2 questions. One, in this series, was it necessary to actually detach? The intramural chords run behind the commissure. Was it necessary to detach in a more significant fashion the commissure to completely unroof the intramural segment and was that associated with an increased risk of insufficiency?

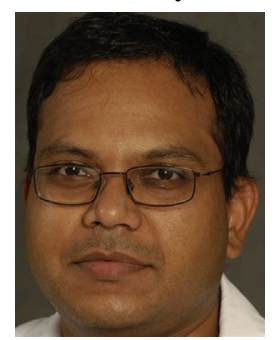

Dr Pranava Sinha (Washington, DC). In this series of 20 patients, no patient required detachment of the commissure. The reason why this is possible is that, as I was mentioning, if you think of the intramural segment as an $\mathrm{O}$, we don't always have to make that unroofing incision at 9 o'clock. If we make that incision at 11 o'clock, so more toward the roof, you are further away from the tip of the commissure, and that's what we have been doing.

As I also mentioned, we excluded patients who had less than 6 months of follow-up. So when we wrapped up this study, if I remember correctly, with 4 or 5 patients, we had to detach the commissure in 1 , which was subsequently reattached, and that patient has a competent aortic valve.

Dr Gaynor. The location of the neo-ostium can be variable within the sinus and occasionally can be close to the commissural post. You discuss moving the stitch a little bit if that's the case. Have you seen any distortion of the valve when it is necessary to do that and have you seen any patients in whom it was not possible to resuspend the commissure because it would compromise the neo-ostium?

Dr Sinha. We all know that the length of the intramural segment is variable, which can actually affect how far your resuspension stitches need to be from the neo-ostium. So I was initially skeptical about moving it a couple of millimeters away, and I was expecting that we are going to end up with distortion of the valve, but surprisingly that is not the case.

We are talking about a 3- or 4-mm coronary ostium with a 6-mm mean intramural length, but the ones with an intramural length of $4 \mathrm{~mm}$, if you just move it by 1 or $2 \mathrm{~mm}$, it works very well.

Dr Gaynor. Have there been any patients in whom you were not able to resuspend using that technique because of potential compromise?

Dr Sinha. There has been 1 patient. Again, he was not included in the series because I actually operated on him just 2 weeks ago. His intramural segment was $4 \mathrm{~mm}$ long. I tried to resuspend the commissure, but there was no way of doing it without compromising the ostium, so I had to leave that alone. I am sure there are going to be a minority of patients in whom you basically have no option.

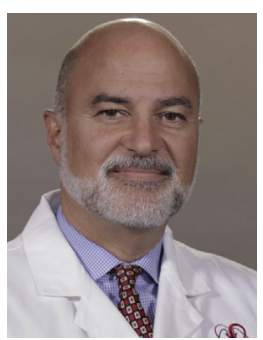

Dr E. Bacha (New York, NY). We recently published a series in the Journal with about 60 patients. Although we agree with your conclusions, our results were more mixed. I am not sure I would agree that "just resuspending the commissure will avoid any AI long term." That's not just what we have seen despite resuspending much like you showed with the same technique. We have had patients, like Bill just mentioned, in whom the neo-ostium is close to the commissure. We have noticed that this area where the tip of the commissure is located has some laxity and abnormal tissue, and therefore it is not normal, at least in this disease. The aortic valve can prolapse easily with CR or not. Have you considered doing a fenestration of the tunnel to the left or the right, depending on which one you are doing, without taking down the commissure?

Dr Sinha. As our data showed, $100 \%$ of our patients had competent aortic valves at 4.3 years of median follow-up. We have not considered doing a fenestration, because it's not possible in every case, and why change a standard procedure if you don't need to? So we have stuck to this modification I would say after the first 9 patients. There are patients in whom it's just going to be impossible to fenestrate because the intramural segment is so short that you cannot do that procedure.

As I mentioned earlier, we firmly believe, and Dr Jonas has this theory, that the commissure area is the least compliant, and it is important to incise through that area. And every one of us who has done this procedure when you are cutting across the intramural segment realizes how tough that tissue is, and it makes sense that that area is not going to allow increased coronary blood flow during times of demand. So we always do a complete unroofing right up to the neoostium and have not done the fenestration procedure.

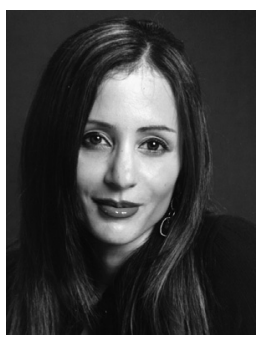

Dr T. Karamlou (Cleveland, Ohio). Nice study. Why did you choose to use mild or greater $\mathrm{AI}$ as an end point versus moderate or severe AI?

Dr Sinha. If you look at the grading of AI by echo, it's none or trivial. Whatever indicators you go by, whether it's the vena contracta, the pressure half time, it only starts as mild, moderate, or greater. And if you see a couple of red cells going across the aortic valve during diastole, that's what we count as trivial. To say this indirectly, any patient who had more than trivial AI was considered as having AI.

Dr Karamlou. I was just questioning the clinical importance of mild versus moderate or severe AI and whether the results are diluted by the inclusion of lesser degrees of regurgitation. 
The second question is that your group looked at a binary end point that was cross-sectional, in other words, the appearance of AI at a certain point in time. As you know, the development of valve deterioration is a time-related event, and there is useful information to be gleaned from the trajectory of deterioration or dysfunction. I am wondering if, in the article, there are some actuarial curves or a longitudinal repeated event analysis to show the trajectories of what the aortic valve is doing, because as you point out in your abstract, the follow-up time was short in many of these patients.

Dr Sinha. We do have those scores in the article, but the important point is that $55 \%$ of these patients who did not have CR had AI at the postoperative echo, the first transesophageal echocardiography that they got, and at the followup that number had gone up to $66 \%$. So it would be safe to assume even with these limited data that this is a progressive pathology. And in every article that's coming out of any center, there are a certain number of patients who require reintervention on their aortic valve. With regard to time to event data, we had a Kaplan-Meier curve in the presentation that is also included in the article.

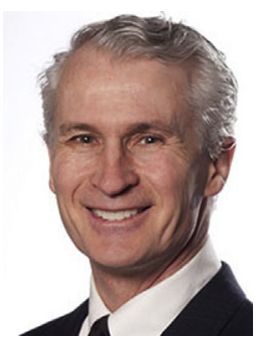

Dr F. Hanley (San Francisco, Calif). I don't have a question. I want to congratulate you on a nice presentation. I think we are all concerned obviously about the aortic valve in this condition. I don't know if I am alone in this concern, but I am also concerned about the exactness of getting these unroofings exactly correct. We all know if you get it $95 \%$ of the way there, it's as if you have done nothing, and if you get it $101 \%$ of the way there, you kind of have a problem.

So based on having done more than 100 of these like that, 2 years ago we moved completely away from unroofing; we don't do it at all. We don't go inside the aorta. We transect the free anomalous coronary outside the aorta, whether it's a left or a right, do an aortic punch in the appropriate sinus at the appropriate spot, and reimplant it directly with 8-0 sutures. Most of these children are 8 to 10 years old or older. Rarely are they younger, but we have performed operations in them at age 3 or 4 years when there is an associated defect like a ventricular septal defect or some other reason we are going in that early anyway.

So we have done probably 25 of these now, combined left and rights. We haven't published it yet because we are waiting 2 years to do 3 -year follow-up imaging, because obviously the other side of the balance here is how well that coronary is going to grow.

The advantages are clear. There is no issue at all when you get the whole intramural length, and, again, I think many in the room if they were honest would say, I' $m$ a little uncomfortable with that unroofing; it just isn't quite an elegant, perfect procedure. And it's never an issue with the AI, and you never open up the aortic root. So I would just put that out there as food for thought for others to consider. 


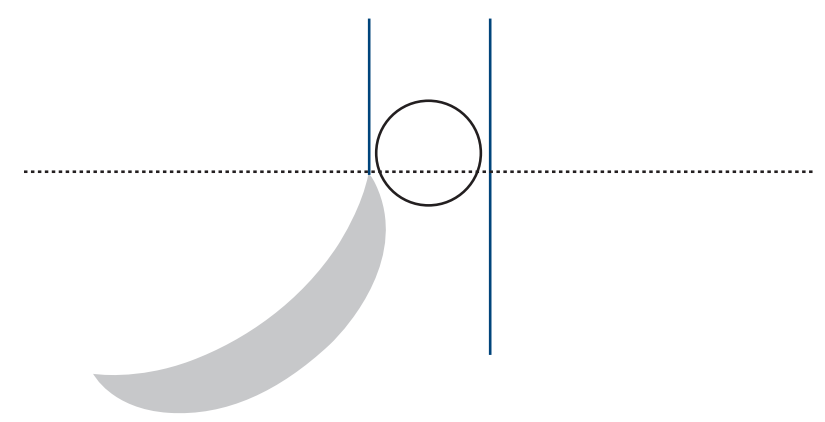

Relationship of the intramural coronary (black circle) to the aortic valve commissure Horizontal dotted line- level of commissural attachment

FIGURE E1. The intramural coronary segment in relation to the commissure is shown. Blue lines represent the aortic wall. Grey: aortic valve cusp.

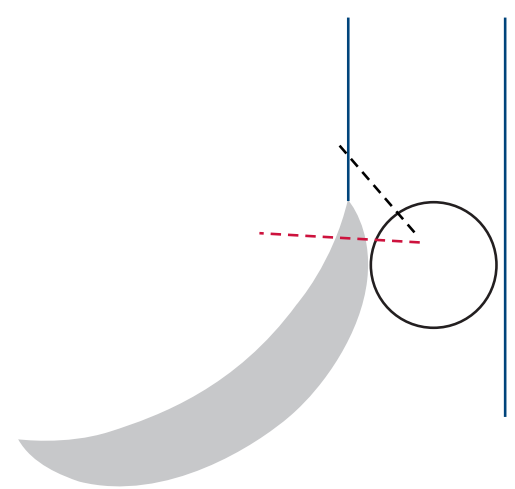

For intramural segments at the level of aortic valve commissure commissural detachment may be avoided by incision along the superior circumference of the vessel at 11'O' clock (black dotted line)

rather than the conventional incision at 9'O' clock (red dotted line)

FIGURE E3. Alternative technique of unroofing in case of the intramural segments at the level of the commissure with avoidance of the commissural detachment. Blue lines represent the aortic wall. Grey: aortic valve cusp.

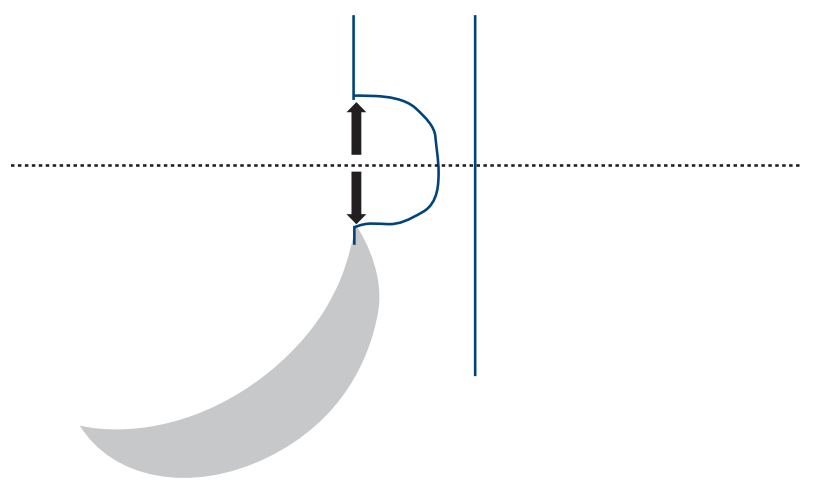

Relationship of the the aortic valve commissure after unroofing in relation to the preoperative level

Horizontal dotted line- preoperative height of commissural attachment

Arrows- direction of elongation

FIGURE E2. The unroofing leads to a change of the level of the commissure in relation to the preoperative level that is lower. Arrows show the direction of the elongation of the aortic wall segment. Blue lines represent the aortic wall. Grey: aortic valve cusp. 

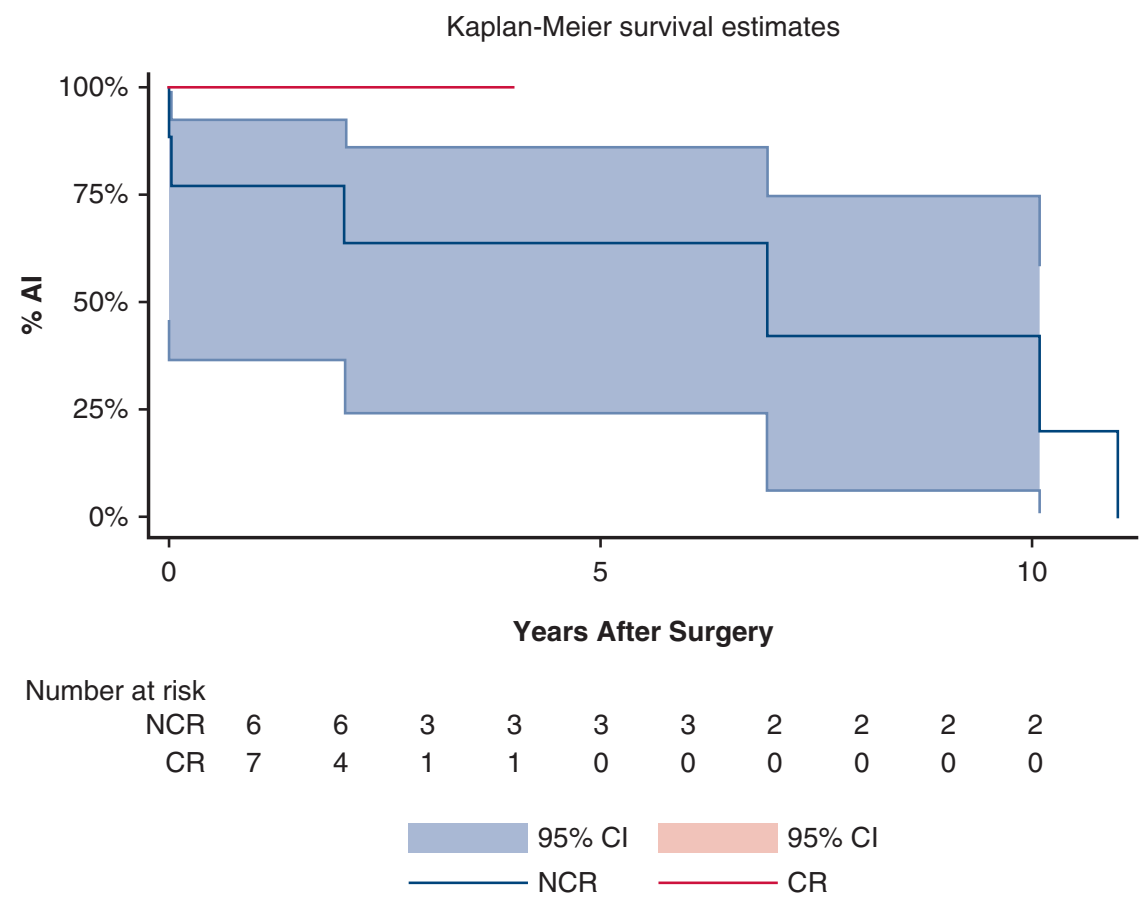

FIGURE E4. Kaplan-Meier curves and 95\% confidence intervals showing freedom from mild or greater aortic regurgitation including all patients (also with $<6$ months follow-up). A log-rank test $(P=.0326)$ demonstrated a greater freedom from mild or greater aortic regurgitation in patients who had a $\mathrm{CR}$ (CR group) compared with those who did not (NCR group). Patients without aortic regurgitation were censored at the time of last known echocardiographic examination. $A I$, Aortic insufficiency; $C I$, confidence interval; $C R$, commissural resuspension; $N C R$, no commissural resuspension. 